\title{
Referral bias in female functional hyperandrogenism and polycystic ovary syndrome
}

\author{
Manuel Luque-Ramírez', Macarena Alpañés ${ }^{1}$, Raul Sanchón ${ }^{1,2}$, \\ Elena Fernández-Durán ${ }^{1}$, Andrés E Ortiz-Flores ${ }^{1}$ and Héctor F Escobar-Morreale' \\ ${ }^{1}$ Diabetes, Obesity and Human Reproduction Research Group, Department of Endocrinology \& Nutrition, Instituto \\ Ramón y Cajal de Investigación Sanitaria IRYCIS, Centro de Investigación Biomédica en Red Diabetes y \\ Enfermedades Metabólicas Asociadas CIBERDEM, Hospital Universitario Ramón y Cajal, Universidad de Alcalá, \\ Carretera de Colmenar km 9'1, E-28034 Madrid, Spain and ${ }^{2}$ Section of Endocrinology and Nutrition, Department of \\ Internal Medicine, Hospital Universitario del Henares, Coslada, Madrid, Spain
}

\author{
Correspondence \\ should be addressed \\ to H F Escobar-Morreale \\ Email \\ hectorfrancisco.escobar@ \\ salud.madrid.org
}

\begin{abstract}
Objective: Women with polycystic ovary syndrome (PCOS) seeking health care in the United States may be more obese and hyperandrogenic than those present in the general population. We aimed to assess the impact of referral bias on European women with functional androgen excess disorders.

Design: Cross-sectional study.

Methods: We studied two groups of patients: i) 368 consecutive patients referred to our clinic for the study of functional hyperandrogenism ( $\mathrm{FH}$ ) (referral patients); ii) 57 consecutive premenopausal patients identified by screening during blood donation (unselected patients). We compared the anthropometric data from the groups of patients with those of two control populations: iii) a group of unselected premenopausal healthy female blood donors (unselected controls); and iv) data available from the local general premenopausal female population.

Results: Referral patients with FH were more hirsute, had a higher percentage of hyperandrogenemia, and fulfilled PCOS criteria more frequently than unselected patients. The prevalence of obesity in unselected controls was similar to that observed in the general population, whereas referral patients and unselected patients were more frequently obese. The prevalence of obesity was also higher among referral patients compared to unselected patients.

Conclusion: Referral bias influences the phenotype of patients with $\mathrm{FH}$. Patients studied at the clinical setting may show more severe hyperandrogenic and obese phenotypes than patients from the general population, even though PCOS appears to be associated with weight excess also in the general European population. This fact should be considered when establishing reference values and control populations for clinical and research purposes.

\section{Introduction}

Obesity has a negative impact on the cardiovascular risk of women with polycystic ovary syndrome (PCOS) by increasing, among others, the risk to develop abnormalities of carbohydrate and lipid metabolism, and blood pressure regulation $(1,2)$. A recent report (3) suggests that US women with PCOS recruited at the clinical setting are more frequently obese, and their hyperandrogenism is
(C) 2015 European Society of Endocrinology Printed in Great Britain more severe than women with PCOS recruited from unselected populations, raising the possibility of referral bias. However, the findings published in this study were obtained from a US multiethnic and multiracial population, and differences in ethnic background and diversity, local environmental factors and access to healthcare resources may also influence the PCOS phenotype $(3,4)$.

Published by Bioscientifica Ltd. 
To our best knowledge, these results have not been confirmed nor refuted in other populations, in particular in Europe. Further studies on this issue, conducted preferably in homogeneous populations, are definitely warranted.

The possibility of referral bias is also of utmost importance for the selection of appropriate controls with the aim of establishing reference values, for both clinical and research purposes. For example, in the case of functional hyperandrogenism (FH) and PCOS the possibility that patients included in clinical series may be more obese than those recruited from the general population (3) may overestimate the risks of obesity and metabolic disorders if controls are selected from the general population instead of being carefully selected to be similar in anthropometric variables.

To provide new insights on these methodological and practical aspects, we conducted an observational crosssectional study including two distinct groups of patients: i) a group of consecutive premenopausal women with $\mathrm{FH}$ referred to our clinic for evaluation of clinical hyperandrogenism or menstrual disorders (referral patients); and ii) a group of premenopausal women with FH identified by screening during a prevalence study (5) previously conducted in unselected blood donors (unselected patients).

Then, we compared the anthropometric data from the groups of patients with those of two control populations: iii) a group of unselected premenopausal healthy female blood donors (unselected controls) recruited during the prevalence study previously mentioned (5); and iv) data available from the local general premenopausal female population (6).

\section{Subjects and methods}

\section{Referral population}

We collected data from 368 consecutive premenopausal women with $\mathrm{FH}$ aged $\geq 18$ years-old referred to our Reproductive Endocrinology clinic from 2004 to 2014 because of symptoms of androgen excess such as hirsutism or menstrual dysfunction (referral patients). Because ovarian ultrasound had not been performed routinely during this period of time, the specific diagnoses comprised by the term FH included classic PCOS, idiopathic hyperandrogenism and idiopathic hirsutism (7). A diagnosis of classic PCOS required the presence of clinical and/or biochemical androgen excess together with ovulatory dysfunction $(8,9)$. Idiopathic hyperandrogenism was defined by the presence of hyperandrogenemia with or without clinical androgen excess in an ovulatory woman (10), and idiopathic hirsutism was defined by the presence of hirsutism in women showing normal serum androgen concentrations and regular ovulatory menstrual cycles (10).

These patients had not received treatment with oral contraceptives, antiandrogenic, or insulin sensitizers during the 6 previous months before enrollment.

\section{Unselected population}

For the present study, we used the data of 241 consecutive unselected women of reproductive age from Spain reporting spontaneously for blood donation to the Blood Bank Facilities of our Hospital and recruited for a prevalence survey of FH previously published (5). After classifying the women according to the same definitions of FH applied to the referral patients, 57 women fulfilling diagnostic criteria of classic PCOS, idiopathic hyperandrogenism or idiopathic hirsutism composed the group of unselected patients, whereas 184 women without any evidence of FH, menstrual dysfunction or history of subfertility served as unselected controls. Regarding unselected patients, women under hormonal contraception were excluded, unless there was a previous and reliable diagnosis of $\mathrm{FH}$, or their clinical history and physical examination were enough to sustain such diagnosis. None of the subjects were younger than 18 years old, which is the minimum legal age for blood donation in Spain. None of the unselected control women had received treatment with oral contraceptives, antiandrogenic, or antidiabetic drugs during the six previous months before enrollment.

\section{Population demographics and other methods}

The counts, distribution and circulating androgen profiles of study subgroups are shown in Table 1 and Supplementary Table 1 (see section on supplementary data given at the end of this article). Both cohorts of women were homogeneous in terms of ethnic and racial origin, most women (99.2\%) were white Mediterranean Caucasians, and showed homogeneous socioeconomic and cultural background because the Spanish National Health System warrants universal healthcare coverage, and both the referral patients and unselected cohorts were recruited at the same city and Institution. None of the women had been previously diagnosed with hypertension, disturbances of carbohydrate metabolism, or cardiovascular disease.

Hirsutism was scored by the same trained physicians in both cohorts, using the modified Ferriman-Gallwey 
Table 1 BMI categories as a function of the phenotypes of patients with functional hyperandrogenism. Data in each category are shown as counts (\%). Percentages were compared by a stratified $\chi^{2}$ test.

\begin{tabular}{|c|c|c|c|c|c|c|}
\hline & \multicolumn{3}{|c|}{$\begin{array}{l}\text { Referral patients (reproductive endocrinology } \\
\text { clinic) }\end{array}$} & \multicolumn{3}{|c|}{$\begin{array}{c}\text { Unselected patients (volunteer blood } \\
\text { donation) }\end{array}$} \\
\hline & Lean & Overweight & Obese & Lean & Overweight & Obese \\
\hline Functional hyperandrogenism & \multicolumn{6}{|c|}{$\chi^{2}=31.8 ; P<0.001$} \\
\hline $\begin{array}{l}\text { Polycystic ovary syndrome (classic } \\
\text { phenotype) }\end{array}$ & $67(29)$ & $58(25)$ & $103(45)$ & $5(25)$ & $10(50)$ & $5(25)$ \\
\hline Idiopathic hyperandrogenism & $46(47)$ & $27(28)$ & $25(26)$ & $8(57)$ & $2(14)$ & $4(29)$ \\
\hline Idiopathic hirsutism & $26(62)$ & $11(26)$ & $5(12)$ & $12(55)$ & $6(27)$ & $4(18)$ \\
\hline & \multicolumn{3}{|c|}{$\chi^{2}=28.1 ; P<0.001$} & \multicolumn{3}{|c|}{$\chi^{2}=4.9 ; P=0.087^{a}$} \\
\hline
\end{tabular}

${ }^{a}$ After merging overweight and obese patients because two cells had expected values $<5$.

method (11). A total score $\geq 8$ was considered abnormal, and hirsutism was graded as mild (mFG 8-14) or moderate to severe $(\mathrm{mFG} \geq 15)(10)$. We recorded BMI $\left(\mathrm{kg} / \mathrm{m}^{2}\right)$ and BMI category (lean (BMI $<25 \mathrm{~kg} / \mathrm{m}^{2}$ ), overweight $\left(25-29.9 \mathrm{~kg} / \mathrm{m}^{2}\right)$ and obesity $\left(\mathrm{BMI} \geq 30 \mathrm{~kg} / \mathrm{m}^{2}\right)$ ).

All women, including patients and controls, did not receive any economic compensation for their participation. All women signed written informed consent and the local Ethics Committee approved the study.

\section{Sampling and assays}

In the group of referral patients, serum and plasma samples were obtained after 12-h overnight fasting, between days 5 and 10 of a spontaneous or progestin induced menstrual bleeding, or at random in amenorrheic patients after excluding pregnancy (2). On the other hand, serum and plasma samples were obtained from women in the unselected group at the time of blood donation, irrespective of meals and day of the menstrual cycle (5). We obtained a sex steroid profile consisting of serum total testosterone, SHBG, androstenedione $\left(\delta_{4}\right)$, and DHEAS concentrations. Total testosterone was measured by direct RIA (Spectria Testosterone RIA, Orion Diagnostica Oy, Espoo, Finland) in the referral patients and by automated immunochemiluminescence in the unselected population (Immulite 2000, Siemens Healthcare Sector, Erlangen, Germany). The lower limits of detection were 0.5 and $0.7 \mathrm{nmol} / 1$ respectively. SHBG, $\delta_{4}$ and DHEAS were measured using automated immunochemiluminescence (Immulite 2000, Siemens Healthcare Sector) with lower limits of detection of $0.02 \mathrm{nmol} / \mathrm{l}$ for SHBG, $1.0 \mathrm{nmol} / 1$ for $\delta_{4}$ and $0.4 \mu \mathrm{mol} / 1$ for DHEAS. The mean intra- and inter-assay CV were $<10 \%$ for all these assays. Free testosterone levels were calculated from total testosterone concentrations and SHBG levels (12). Hyperandrogenemia was defined by total testosterone, free testosterone, $\delta_{4}$, or DHEAS concentrations above the 95th percentile of our in-house reference range obtained from premenopausal nonhyperandrogenic women with regular menses (13) in the case of referral patients, or from the unselected control group of volunteer donors in the case of unselected patients. Secondary causes of hirsutism or ovulatory dysfunction were excluded by means of either clinical exam or appropriate laboratory testing. All of the assays were run at the time of subjects' recruitment.

\section{Statistical analysis}

Data are shown as mean \pm s.D., 95\% CI and counts (\%), as indicated. For continuous variables, logarithmic transformation was applied as needed to ensure normality as needed according to the results of the KolmogorovSmirnov test. The modified hirsutism score was compared among groups of patients by Mann-Whitney $U$ test. Age was compared between both groups of patients and unselected controls by Kruskal-Wallis $H$ test. The BMI of the subgroups was submitted to univariate one-way general linear model in which referral patients, unselected patients or unselected control status was introduced as independent variable, and age was introduced as covariate. The mean effects of the independent variable were analyzed applying the Bonferroni correction for multiple comparisons. The distribution of FH diagnoses, grade of hirsutism, hyperandrogenemia, and BMI categories between referral and unselected patients were compared by $\chi^{2}$ test.

The odds ratios $(95 \% \mathrm{CI})$ for obesity in the group of referral patients and unselected patients with respect to the group of unselected controls were calculated by logistic binary regression. We also calculated the odds 

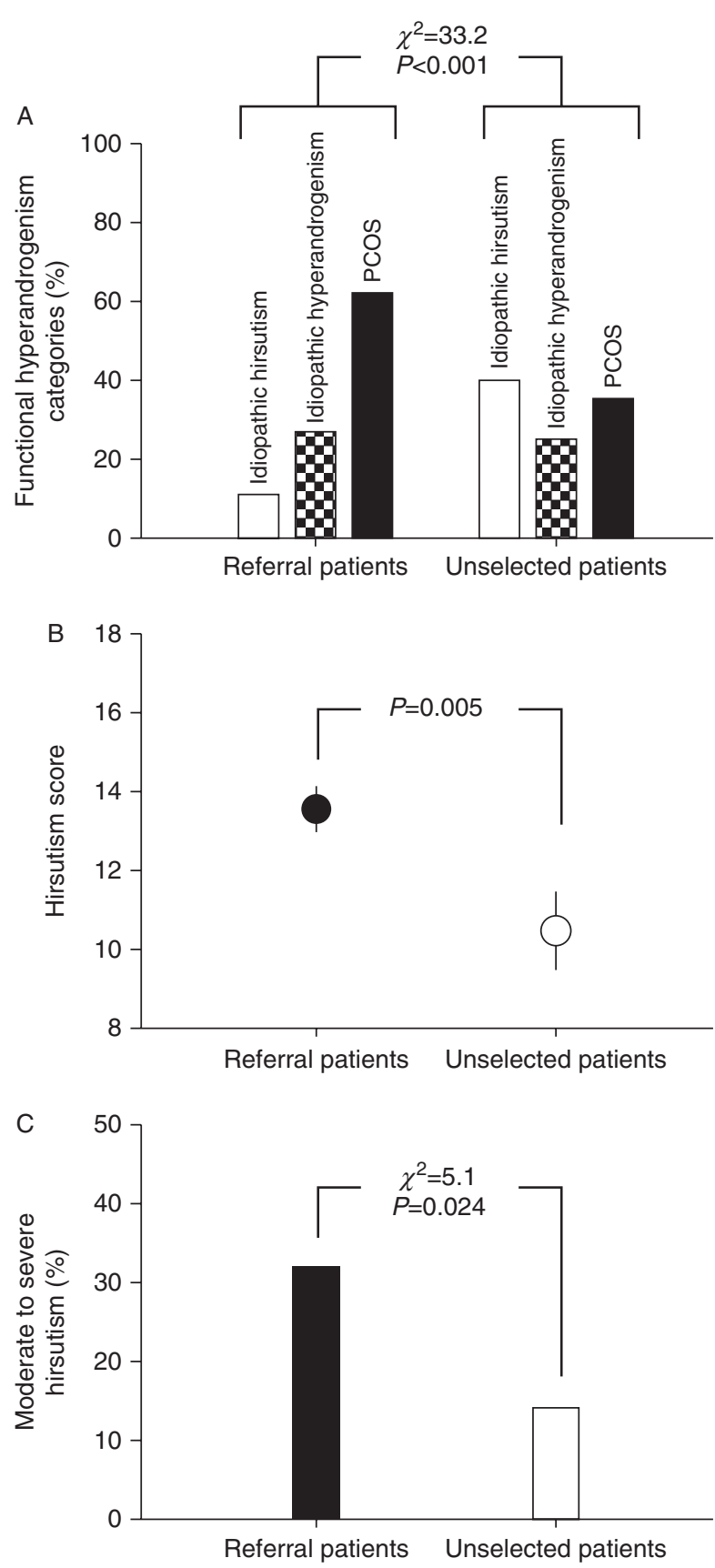

\section{Figure 1}

Distribution of functional hyperandrogenism categories and hyperandrogenic features as a function of study subgroups. (A) Percentage of women matching the different diagnostic categories of functional hyperandrogenism. Data are percentages. The differences were analyzed by stratified $\chi^{2}$ test. (B) Modified Ferriman-Gallwey score. Data are means and $95 \% \mathrm{Cls}$ and were compared by Mann-Whitney $U$ test. (C) Percentage of women with moderate or severe hirsutism (modified Ferriman-Gallwey score $\geq 15$ ). The differences were analyzed by stratified $\chi^{2}$ test. ratios (95\% CI) for obesity in each category with respect to published data regarding their prevalence in women aged 18-45 years from Madrid (6) using the Newcombe-Wilson method without continuity correction (14).

We used the IBM Statistical Package for the Social Sciences Statistics 20 for analyses. $P<0.05$ was considered statistically significant.

\section{Results \\ Impact of referral bias on FH phenotype}

The hyperandrogenic phenotype was more severe in referral patients, with PCOS being the most frequent specific diagnosis in them, whereas the more frequent $\mathrm{FH}$ diagnosis in unselected patients was idiopathic hirsutism (Fig. 1A). Among hirsute patients, the hirsutism score was higher in referral patients compared with patients in the unselected cohort (Fig. 1B), even though the percentage of patients with hirsutism was the same in both cohorts ( $80.2 \%$ vs $80.8 \%, \chi^{2}=0.064, P=0.801$ respectively).

Likewise, the percentage of hirsute patients with moderate-severe grade ( $\mathrm{mFG}$ score $\geq 15$ ) was higher in referral patients with respect to unselected patients (Fig. 1C). In conceptual agreement, patients from the clinical population had higher circulating total testosterone, free testosterone, $\delta_{4}$, and DHEAS concentrations than unselected patients (Supplementary Table 1), although we did not make direct comparisons between the referral and unselected groups because of the different hormonal assays used for serum testosterone measurement. Also, serum and plasma samples were obtained from women in the unselected cohort at the time of blood donation, irrespective of menstrual cycle phase.

Nonetheless, the percentage of referral patients with elevated free testosterone and $\delta_{4}$ levels were higher in the clinical population than in unselected patients (Fig. 2).

\section{Impact of referral bias on BMI and BMI categories}

When evaluating the distribution of $\mathrm{FH}$ phenotypes among BMI categories in referral and unselected patients, we found significant differences that consisted mainly of a greater proportion of patients with PCOS and obesity in the referral cohort compared to the cohort of unselected patients (Table 1).

Since unselected control women were older than both referral patients and unselected patients ( $33 \pm 8$ vs $26 \pm 6$ vs $27 \pm 7$ years, respectively, $P<0.001$ ), we introduced age as covariate in further analyses. Both groups of patients 

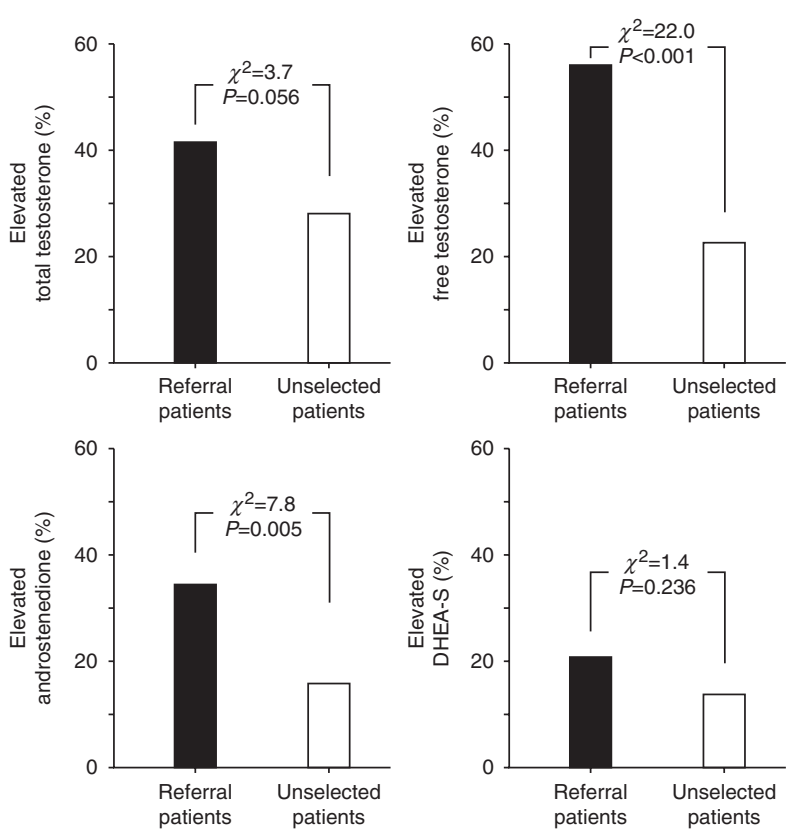

Figure 2

Percentage of patients with hyperandrogenemia as a function of study subgroups. Data are percentages. The differences were analyzed by stratified $\chi^{2}$ test.

had higher mean BMIs than unselected controls, but referral and unselected patients showed similar BMI values (Fig. 3A).

Regarding BMI categories, the distribution of unselected controls was similar to the estimates for women from Madrid's general population aged 18-44 year-old (6), most of whom were lean (Fig. 3B). The odds for obesity were increased in referral patients and in unselected patients, with respect to the general premenopausal female population of Madrid (Fig. 3B). In addition, referral patients had a twofold risk in presenting with obesity compared to unselected patients (OR: $2.1(1.1-4.1)$ ).

\section{Discussion}

In agreement with the previous report from US populations (3), our present results indicate the existence of referral bias in the phenotype of women seeking treatment for FH at a Reproductive Endocrinology Clinic in Spain.

First, the patients referred for evaluation to our clinic had a more severe hyperandrogenic phenotype compared with unselected women from the general population found to have FH by screening after reporting spontaneously for blood donation.
Patients at the clinical setting were more hirsute, had a higher hirsutism grade, presented more frequently with hyperandrogenemia, and suffered classic PCOS more frequently than their unselected counterparts recruited directly from the general population. Actually, these
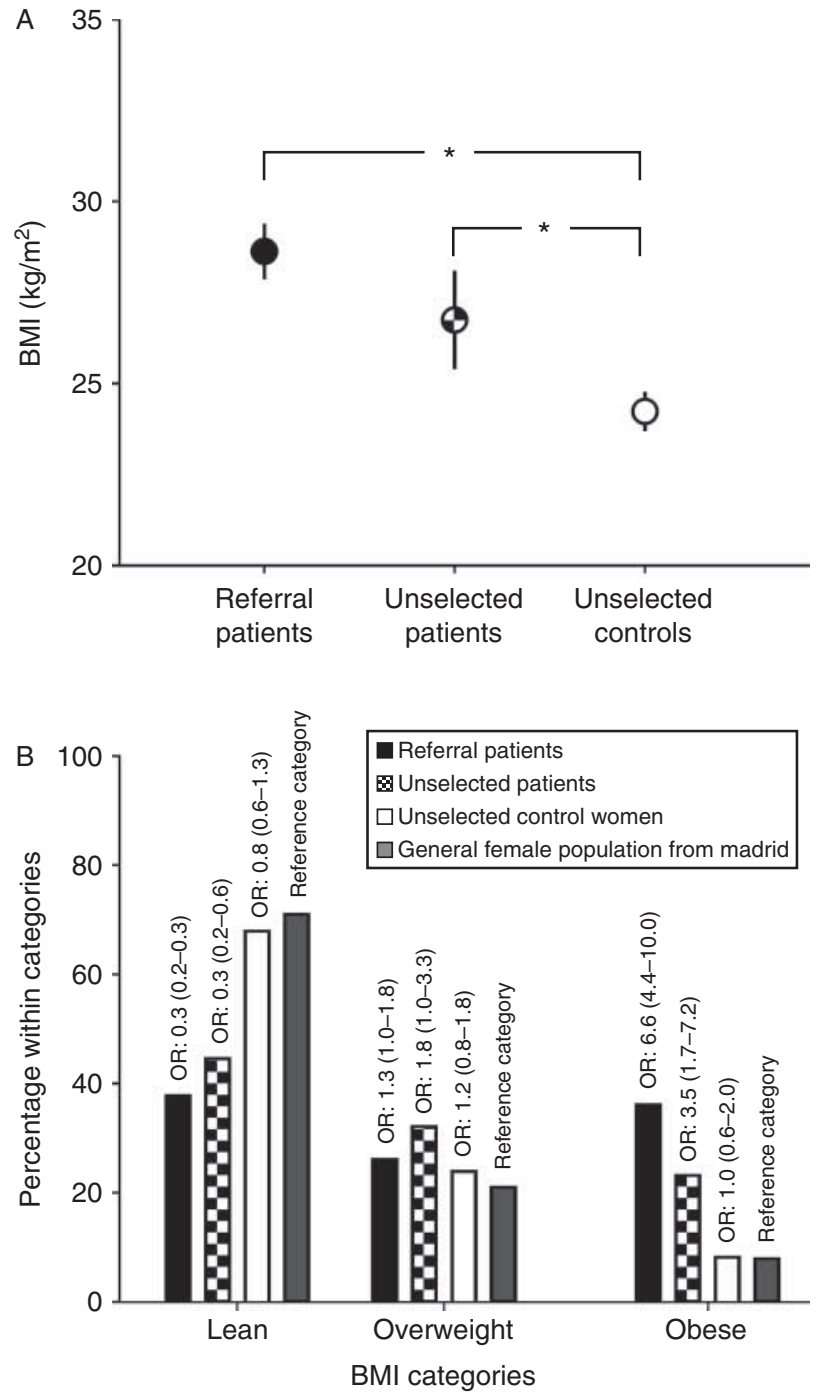

Figure 3

$\mathrm{BMI}$ and $\mathrm{BMI}$ categories. (A) BMI as a function of patient subgroups and unselected controls. Data are means and $95 \% \mathrm{Cls}$. Comparisons were performed by a general lineal model introducing age as a covariate. *, statistically significant difference at the $P<0.05$ level. (B) Distribution of patients' subgroups, unselected controls and premenopausal women from the general population as a function of BMI categories. The odds ratios and $95 \%$ Cls for obesity in each category were calculated by the Newcombe-Wilson method without continuity correction using as a reference category the published data obtained from women aged 18-45 years from Madrid, Spain. 
findings are not surprising because both hirsutism and menstrual disorders have a considerable negative impact on the health related quality of life (HRQoL) of women with hyperandrogenic disorders (15), and might prompt patients to seek appropriate healthcare more actively than women with milder hyperandrogenic symptoms (3).

The presence of a more severe hyperandrogenic phenotype in patients referred to our Reproductive Endocrinology clinic has practical implications in this setting. On the one hand, hyperandrogenemia may predict the metabolic phenotype of these women (16) by increasing the incidence of dysglycemia (16), impaired blood pressure regulation (17), or subclinical atherosclerosis (18), independently of obesity. On the other hand, the classic hyperandrogenic and anovulatory PCOS phenotype that was particularly prevalent in referral patients is associated with insulin resistance and metabolic dysfunction more frequently than with ovulatory phenotypes, even after adjusting by fat mass (19). Furthermore, patients with classic PCOS may show an increased risk for the development of subclinical atherosclerosis compared with patients suffering ovulatory and nonhyperandrogenic PCOS phenotypes (20).

Second, our data suggest that weight excess is intimately associated with $\mathrm{FH}$ in both referral and unselected women from Spain, even though such association may be partly the result of referral bias as occurs in the US population (3). In our study, obesity was associated with hyperandrogenism not only in patients in the referral cohort - who might have been referred more frequently to our Clinic considering the profound impact that obesity has on the HRQoL of PCOS patients (21) - but also in patients reporting spontaneously for blood donation. In agreement, patients in both cohorts had increased mean BMI values and frequencies of obesity compared with premenopausal women from the general population. But in addition, we found a higher prevalence of obesity in referral patients, particularly in those women with classic phenotype, compared with their unselected counterparts. As proposed by Ezeh et al. (3) for the US population, these differences may be related to a more severe ovulatory dysfunction in obese patients (22), which may prompt these women to seek medical care actively. Adiposity and obesity not only facilitate androgen excess in predisposed women (23), but is also a major contributor to the insulin resistance and cardiometabolic comorbidities of women with androgen excess $(1,24)$.

Nonetheless, we also found higher odds for obesity among referral patients with respect to our cohort of unselected controls and to the data available from the general population than in the previous US study (3). Moreover, the odds were closer to those reported for Caucasians in a recent meta-analysis describing the prevalence of obesity in PCOS (25).

The differences between our present study and the previous US report may be explained by: i) we studied a homogeneous population in terms of ethnic background (over 99\% of these women were white Mediterranean Caucasians), and ii) the higher prevalence of obesity in the US general premenopausal female population with respect to the general population from Spain. Of note, the unselected cohort of control women in our study was certainly representative of the premenopausal women in our general population, because their prevalence of weight excess disorders was the same than that reported for women aged 18-45 years old living in Madrid (6).

In our population, the gap between referral patients and unselected patients in terms of mean BMI was smaller than that in the US population (3). Referral women with PCOS from Alabama showed markedly higher mean BMI values than unselected patients, who showed mean BMI values similar to those of unselected controls (3). On the contrary, we found no significant differences between referral and unselected patients in mean BMI values, and our unselected patients identified by screening had higher mean BMI values compared with unselected controls. Bearing in mind that overweight and obese women from Spain have a $28.3 \%$ prevalence of PCOS (26), self or otherwise referral patients with FH are likely to reflect the BMI picture of that disorder in our general population.

These discrepancies may be also explained by differences in the access to healthcare resources between Spain and the United States. The Spanish National Health System warrants universal healthcare coverage facilitating that women with mild dermo-cutaneous disturbances related to FH or weight excess sought for medical care. On the contrary, only women with more severe phenotypes might seek medical advice in the United States considering the more restrictive access to the insurancebased US health system.

Our study, however, was not free of limitations: i) the sampling conditions and immunoassays used to measure serum total testosterone concentrations were different in the referral patients and unselected cohorts, yet we established the upper limits of normality for each cohort separately. Noteworthy, when the ability of the immunoassay used in the preset study to make out between hyperandrogenic and normoandrogenic women has 
been tested, the percentage of samples with abnormal testosterone concentrations was comparable to that observed when isotope-dilution gas chromatographymass spectrometry upper limit was applied (27); ii) because ovarian ultrasound was not performed routinely, we cannot determine how many patients with idiopathic hyperandrogenism and idiopathic hirsutism fulfilled recent definitions of PCOS (28). Therefore, we cannot extrapolate any conclusion to women presenting with normoandrogenic PCOS phenotypes, yet the presence of polycystic ovarian morphology does not appear to confer any extra cardiometabolic risk to women with androgen excess (29); and iii) sampling in the unselected cohort was conducted in non-fasting conditions, and we have not been able to study variables such as carbohydrate metabolism, insulin resistance and lipid profiles that require fasting to be correctly assessed.

In conclusion, the possibility of referral bias should be considered when analyzing the phenotype of androgen excess disorders. Patients studied in the clinical setting represent a more severe hyperandrogenic and metabolically morbid subpopulation than those present in the general population. Obesity appears to be an intrinsic association of FH and PCOS, at least in women homogeneous in terms of race, socioeconomic conditions, access to healthcare facilities and ethnicity such as the Mediterranean Spaniards studied here. Finally, women with PCOS at the clinical setting make up a particular subset of patients at cardiometabolic risk because their odds for obesity are even higher that those of patients from the general population. Thus, a comprehensive cardiometabolic assessment is likely warranted in this population.

\section{Supplementary data}

This is linked to the online version of the paper at http://dx.doi.org/10.1530/ EJE-15-0646.

\section{Declaration of interest}

The authors declare that there is no conflict of interest that could be perceived as prejudicing the impartiality of the research reported.

\section{Funding}

This study was supported by grants PI1100357 and PI1400649 from Instituto de Salud Carlos III, Spanish Ministry of Economy and Competitiveness. Centro de Investigación Biomédica en Red Diabetes y Enfermedades Metabólicas Asociadas is also an initiative of Instituto de Salud Carlos III. Supported in part by funds from Fondo Europeo de Desarrollo Regional (European Union).

\section{Author contribution statement}

M Luque-Ramírez and $\mathrm{H} F$ Escobar-Morreale designed the study, researched data, analyzed the results and wrote the final version of the manuscript. M Alpañés, R Sanchón, A E Ortiz-Flores and E Fernández-Durán researched data, drafted the manuscript and contributed critically to discussion. All authors approved the decision to submit the manuscript and take public responsibility for its whole content.

\section{References}

1 Lim SS, Norman RJ, Davies MJ \& Moran LJ. The effect of obesity on polycystic ovary syndrome: a systematic review and meta-analysis. Obesity Reviews 2013 1495-109. (doi:10.1111/j.1467-789X.2012.01053.x)

2 Luque-Ramirez M, Marti D, Fernandez-Duran E, Alpanes M, AlvarezBlasco F \& Escobar-Morreale HF. Office blood pressure, ambulatory blood pressure monitoring, and echocardiographic abnormalities in women with polycystic ovary syndrome: role of obesity and androgen excess. Hypertension 201463 624-629. (doi:10.1161/ HYPERTENSIONAHA.113.02468)

3 Ezeh U, Yildiz BO \& Azziz R. Referral bias in defining the phenotype and prevalence of obesity in polycystic ovary syndrome. Journal of Clinical Endocrinology and Metabolism 201398 E1088-E1096. (doi:10.1210/jc. 2013-1295)

4 Zhao Y \& Qiao J. Ethnic differences in the phenotypic expression of polycystic ovary syndrome. Steroids $2013 \mathbf{7 8} 755-760$. (doi:10.1016/ j.steroids.2013.04.006)

5 Sanchon R, Gambineri A, Alpanes M, Martinez-Garcia MA, Pasquali R \& Escobar-Morreale HF. Prevalence of functional disorders of androgen excess in unselected premenopausal women: a study in blood donors. Human Reproduction 201227 1209-1216. (doi:10.1093/humrep/des028)

6 Ruiz Moreno E, del Pozo de la Calle S, Cuadrado Vives C, Valero Gaspar T, Ávila Torres JM, Belmonte Cortés S \& Varela Moreiras G. Encuesta de nutrición de la Comunidad de Madrid (ENUCAM). Madrid, Spain: Comunidad de Madrid, 2014. (available at: http://www. observatorio.naos.aesan.msssi.gob.es/docs/docs/documentos/ ENUCAM_2014.pdf)

7 Escobar-Morreale HF, Sanchon R \& San Millan JL. A prospective study of the prevalence of nonclassical congenital adrenal hyperplasia among women presenting with hyperandrogenic symptoms and signs. Journal of Clinical Endocrinology and Metabolism 200893 527-533. (doi:10.1210/jc.2007-2053)

8 Azziz R, Carmina E, Dewailly D, Diamanti-Kandarakis E, EscobarMorreale HF, Futterweit W, Janssen OE, Legro RS, Norman RJ, Taylor AE et al. The androgen excess and PCOS society criteria for the polycystic ovary syndrome: the complete task force report. Fertility and Sterility 200991 456-488. (doi:10.1016/j.fertnstert.2008.06.035)

9 National Institutes of Health. Evidence-based methodology workshop on polycystic ovary syndrome. Bethesda, MD, USA: NIH, 2012. (available at: https://prevention.nih.gov/docs/programs/pcos/ FinalReport.pdf)

10 Escobar-Morreale HF, Carmina E, Dewailly D, Gambineri A, Kelestimur F, Moghetti P, Pugeat M, Qiao J, Wijeyaratne CN, Witchel SF et al. Epidemiology, diagnosis and management of hirsutism: a consensus statement by the androgen excess and polycystic ovary syndrome society. Human Reproduction Update 201218 146-170. (doi:10.1093/humupd/dmr042)

11 Hatch R, Rosenfield RL, Kim MH \& Tredway D. Hirsutism: implications, etiology, and management. American Journal of Obstetrics and Gynecology 1981140 815-830.

12 Vermeulen A, Verdonck L \& Kaufman JM. A critical evaluation of simple methods for the estimation of free testosterone in serum. Journal of Clinical Endocrinology and Metabolism 199984 3666-3672. (doi:10.1210/jcem.84.10.6079) 
13 Alpanes M, Luque-Ramirez M, Martinez-Garcia MA, FernandezDuran E, Alvarez-Blasco F \& Escobar-Morreale HF. Influence of adrenal hyperandrogenism on the clinical and metabolic phenotype of women with polycystic ovary syndrome. Fertility and Sterility 2015103 795-801 e792. (doi:10.1016/j.fertnstert.2014.12.105)

14 Newcombe RG. Two-sided CIs for the single proportion: comparison of seven methods. Statistics in Medicine 199817 857-872. (doi:10.1002/ (SICI) 1097-0258(19980430)17:8<857::AID-SIM777 > 3.0.CO;2-E)

15 Cinar N, Harmanci A, Demir B \& Yildiz BO. Effect of an oral contraceptive on emotional distress, anxiety and depression of women with polycystic ovary syndrome: a prospective study. Human Reproduction 201227 1840-1845. (doi:10.1093/humrep/des113)

16 O'Reilly MW, Taylor AE, Crabtree NJ, Hughes BA, Capper F, Crowley RK, Stewart PM, Tomlinson JW \& Arlt W. Hyperandrogenemia predicts metabolic phenotype in polycystic ovary syndrome: the utility of serum androstenedione. Journal of Clinical Endocrinology and Metabolism 201499 1027-1036. (doi:10.1210/jc.2013-3399)

17 Chen MJ, Yang WS, Yang JH, Chen CL, Ho HN \& Yang YS. Relationship between androgen levels and blood pressure in young women with polycystic ovary syndrome. Hypertension 200749 1442-1447. (doi:10.1161/HYPERTENSIONAHA.106.083972)

18 Luque-Ramirez M, Mendieta-Azcona C, Alvarez-Blasco F \& Escobar-Morreale HF. Androgen excess is associated with the increased carotid intima-media thickness observed in young women with polycystic ovary syndrome. Human Reproduction 200722 3197-3203. (doi:10.1093/humrep/dem324)

19 Moghetti P, Tosi F, Bonin C, Di Sarra D, Fiers T, Kaufman JM, Giagulli VA, Signori C, Zambotti F, Dall'Alda M et al. Divergences in insulin resistance between the different phenotypes of the polycystic ovary syndrome. Journal of Clinical Endocrinology and Metabolism 2013 98 E628-E637. (doi:10.1210/jc.2012-3908)

20 Calderon-Margalit R, Siscovick D, Merkin SS, Wang E, Daviglus ML, Schreiner PJ, Sternfeld B, Williams OD, Lewis CE, Azziz R et al. Prospective association of polycystic ovary syndrome with coronary artery calcification and carotid-intima-media thickness: the Coronary Artery Risk Development in Young Adults Women's study. Arteriosclerosis, Thrombosis, and Vascular Biology 201434 2688-2694. (doi:10.1161/ATVBAHA.114.304136)
21 Alvarez-Blasco F, Luque-Ramirez M \& Escobar-Morreale HF. Obesity impairs general health-related quality of life (HR-QoL) in premenopausal women to a greater extent than polycystic ovary syndrome (PCOS). Clinical Endocrinology 201073 595-601. (doi:10.1111/j.13652265.2010.03842.x)

22 Liou TH, Yang JH, Hsieh CH, Lee CY, Hsu CS \& Hsu MI. Clinical and biochemical presentations of polycystic ovary syndrome among obese and nonobese women. Fertility and Sterility 200992 1960-1965. (doi:10.1016/j.fertnstert.2008.09.003)

23 Escobar-Morreale HF \& San Millan JL. Abdominal adiposity and the polycystic ovary syndrome. Trends in Endocrinology and Metabolism 2007 18 266-272. (doi:10.1016/j.tem.2007.07.003)

24 Randeva HS, Tan BK, Weickert MO, Lois K, Nestler JE, Sattar N \& Lehnert H. Cardiometabolic aspects of the polycystic ovary syndrome. Endocrine Reviews 201233 812-841. (doi:10.1210/er.20121003)

25 Lim SS, Davies MJ, Norman RJ \& Moran LJ. Overweight, obesity and central obesity in women with polycystic ovary syndrome: a systematic review and meta-analysis. Human Reproduction Update 201218 618-637. (doi:10.1093/humupd/dms030)

26 Alvarez-Blasco F, Botella-Carretero JI, San Millan JL \& EscobarMorreale HF. Prevalence and characteristics of the polycystic ovary syndrome in overweight and obese women. Archives of Internal Medicine 2006166 2081-2086. (doi:10.1001/archinte.166.19.2081)

27 Taieb J, Mathian B, Millot F, Patricot MC, Mathieu E, Queyrel N, Lacroix I, Somma-Delpero C \& Boudou P. Testosterone measured by 10 immunoassays and by isotope-dilution gas chromatography-mass spectrometry in sera from 116 men, women, and children. Clinical Chemistry 200349 1381-1395. (doi:10.1373/49.8.1381)

28 Andreani CL, Pierro E, Lanzone A, Lazzarin N, Capitanio G, Giannini P $\&$ Mancuso S. Effect of gonadotropins, insulin and IGF I on granulosa luteal cells from polycystic ovaries. Molecular and Cellular Endocrinology 1994106 91-97. (doi:10.1016/0303-7207(94)90190-2)

29 Dewailly D, Lujan ME, Carmina E, Cedars MI, Laven J, Norman RJ \& Escobar-Morreale HF. Definition and significance of polycystic ovarian morphology: a task force report from the Androgen Excess and Polycystic Ovary Syndrome Society. Human Reproduction Update 2014 20 334-352. (doi:10.1093/humupd/dmt061) 